\title{
Mechanical stretching promotes the differentiation of BMSC into pelvic floor ligament fibroblasts
}

\author{
Bing Zhao ${ }^{1, *}$, Junmin Wang ${ }^{2,1, *}$, Chenchen Ren ${ }^{1}$, Mengcai Hu${ }^{1}$, Huiyan Wu ${ }^{1}$, Lulu \\ Chen ${ }^{1}$, Xiaojun Liu ${ }^{3}$, Luwen Wang ${ }^{1}$, Feng $\mathrm{Xu}^{1}{ }^{1}$, Xueqin Zheng ${ }^{1}$, Juan Chen ${ }^{1}$ and \\ Shihong Cui ${ }^{1}$

\footnotetext{
${ }^{1}$ Third Affiliated Hospital of Zhengzhou University, Zhengzhou, Henan Province 450052, China

${ }^{2}$ Department of Human Anatomy, Basic Medical College of Zhengzhou University, Zhengzhou, Henan Province 450052, China

${ }^{3}$ Henan Medical Equipment Inspection Institute, Zhengzhou, Henan Province 450052, China

*These authors have contributed equally to this work
} \\ Correspondence to: Shihong Cui, email: shihongcui@126.com
}

Keywords: mechanical stretch; BMSC; KDM4B; LOX; elastin

Received: December 28, $2016 \quad$ Accepted: July 24, $2017 \quad$ Published: February 07, 2018

Copyright: Zhao et al. This is an open-access article distributed under the terms of the Creative Commons Attribution License 3.0 (CC BY 3.0), which permits unrestricted use, distribution, and reproduction in any medium, provided the original author and source are credited.

\section{ABSTRACT}

Pelvic floor dysfunction (PFD) is a prevalent and debilitating condition in aging women that is associated with weakened pelvic connective tissue. Fibroblasts are the main component of pelvic connective tissue and play a vital role in the maintenance of the pelvic organ. Mechanical stretching is a known modulator leading to the proliferation and differentiation of bone marrow mesenchymal stem cells (BMSCs). In this paper, we measured the lysyl oxidase (LOX), elastin and KDM4B expression in cultured BMSCs under different cyclic mechanical stretch (CMS) conditions via Western blot assays. Our findings indicated that the expression of these proteins was significantly higher in the CMS group than in the normal control group, and $10 \%$ CMS at a $0.5 \mathrm{~Hz}$ frequency had the best stimulatory effect. We further evaluated the influence of KDM4B on LOX and elastin expression via knockdown and overexpression of KDM4B in BMSCs. Chromatin immunoprecipitation (ChIP)-PCR analysis was performed to determine how $\mathrm{H} 3 \mathrm{~K} 9 \mathrm{me} 3$ and $\mathrm{KDM} 4 \mathrm{~B}$ affect the regulatory regions of LOX and elastin in BMSCs subjected to $10 \%$ CMS combined with KDM4B knockdown. Finally, we verified the expression of KDM4B under CMS in vivo by subcutaneously transplanting KDM4B- overexpressing BMSCs into mice via immunofluorescence assays. The results showed that activating KDM4B by mechanical stretching increased the protein and gene expression levels of LOX and elastin and induced more LOX-positive cells in the BMSCs. These data suggest that CMS contributes to the differentiation of mesenchymal stem cells (MSCs) into fibroblasts, which indicates its potential use as a cell-based therapy for PFD.

\section{INTRODUCTION}

Pelvic floor dysfunction (PFD), mainly including stress urinary incontinence (SUI), pelvic organ prolapse (POP), and fecal incontinence (FI), is common in women and more prevalent in the aging population [1-3]. PFD is a complex disease that involves many factors, and the majority of research suggests that the total collagen content is decreased in pelvic connective tissue and that the pelvic support tissues (the ligament, muscle and fascia) are relaxed because of reduced collagen fibers, which eventually leads to PFD $[4,5]$. Although PFD is not a lifethreating disease, it causes urinary and fecal incontinence, dysuria, obstructed defecation, abdominal straining, and sexual dysfunction, which may affect a patient's physical and mental wellbeing as well as their health and quality of life. 
Current treatment options include surgery, conservative drug therapy and rehabilitation treatment, such as pelvic floor muscle training; these treatments have been shown to be effective but defective [6]. Damage to ligament tissues enables the formation of scar tissue that does not undergo regeneration during the repair process. Moreover, the occurrence and development of PFD is closely related to the structural function of the uterosacralcardinal ligament, which provides the main support for the pelvic floor. Therefore, methods of restoring the normal structure and function to damaged uterine ligaments must be developed to resolve the issues associated with PFD.

Bone marrow mesenchymal stem cells (BMSCs) are among the best characterized sources of stem cells, and they exhibit active self-proliferative and multi-lineage differentiation abilities and have the potential to develop into adipocytes, osteocytes or chondrocytes and cells of other embryonic lineages $[7,8]$. Furthermore, BMSCs can secrete bioactive factors that improve tissue repair [9]. Therefore, BMSCs hold great promise for PFD treatment. When tissues or cells are damaged, both endogenous and exogenous BMSCs will transfer to the injured area and promote repair functions. BMSCs applied via periurethral injection have been reported to restore damaged external urethral sphincters and significantly improve SUI symptoms in animal models of SUI [10]. BMSCs have been observed at the site of injury of damaged ligaments during the ligament injury repair process, and they appeared similar to the surrounding ligament cells [11]. Recent studies have focused on MSC "plasticity" in terms of differentiation, which is based on the capacity of MSCs to differentiate into mature cells that differ from their original tissue; this differentiation is achieved through cell cultivation in differential media and identified by evaluating the extent of differentiation [12].

Elastic fiber is an important component of pelvic connective tissue and has excellent flexibility and variability. Elastic protein (elastin) is a core protein of elastic fiber located in the pelvic floor extracellular matrix (ECM) [13]. Reduced synthesis and changes in the structure of elastin can promote the occurrence of POP. Lysyl oxidase (LOX), a copper-dependent monoamine oxidase, is a key enzyme that acts on the ECM, and it is involved in collagen and elastin synthesis in local tissues $[14,15]$. Histone is involved in the epigenetic machinery, and it has a significant role in determining the fate of MSCs by removing certain factors from lineage-specific genes to promote differentiation towards a particular lineage [16]. The histone methylation of lysine and arginine is controlled by the actions of methylases and demethylases [17]. KDM4B (Jmjd2b), a recently recognized member of the histone lysine demethylase Jmjd2 family, contains the catalytic Jumonji C (JmjC) domain $[18,19]$ and has the potential to facilitate the differentiation of BMSCs towards a particular lineage. Therefore, KDM4B likely plays an important role in the direction of BMSC differentiation and tissue regeneration. KDM4B specifically removes histone $3 \mathrm{~K} 9$ trimethylation ( $\mathrm{H} 3 \mathrm{~K} 9 \mathrm{me} 3$ ), an epigenetic methylation mark associated with gene repression and target gene expression regulation. KDM4B is closely involved in many biological processes, such as transcriptional regulation, heterochromatin formation and homeotic gene silencing [20, 21]. Recently, mounting evidence has revealed an epigenetic role of KDM4B in stem cell differentiation and inflammation [22].

Cyclic mechanical stretch (CMS) performs an important action in maintaining the steady state of cells. Under physiological conditions, connective tissue components will change if the ligament tissues are under a certain tension load for a long period of time [23]. At the cellular level, a mechanical signal alters the cell morphology and differentiation, skeleton structure and gene expression. Furthermore, the synthesis and secretion of the ECM is regulated by mechanical stimulation through transcription, post-translational modification, etc. [24-26]. Zhang et al. found that cyclic mechanical stimulation promoted migration but inhibited the invasion of rat bone marrow stromal cells [27].

Our previous research suggested that under mechanical tension stimulation, the indirect co-culture of pelvic floor ligament fibroblast cells and rat BMSCs in vitro could induce the differentiation of BMSCs into pelvic floor ligament fibroblasts that exhibit increased expression of elastin, LOX, and fibulin-5; these fibroblasts were able to effectively repair the PFD caused by damaged pelvic ligaments, fascia, muscles and other support tissue [28]. In this study, we investigated the effect of mechanical force on the oriented differentiation of BMSCs into fibroblasts in vitro and in vivo and studied the mechanism by which mechanical loading epigenetically regulates gene expression during differentiation. We found that at $10 \%$ CMS and $0.5 \mathrm{~Hz}$ cyclic stretching, BMSC differentiation was increased via an increased release of LOX and elastin, which was promoted by the activity of KDM4B and H3K9me3. These findings indicate that mechanical stretching could represent a possible strategy for manipulating BMSCs towards differentiation into ligament fibroblasts and suggest that this technique could be beneficial as a cell therapy approach for managing PFD.

\section{RESULTS}

\section{Protein levels of elastin, LOX and KDM4B in the BMSCs with different CMS extensions}

Western blot assays were used to evaluate the effects of $1 \%, 2 \%, 4 \%$ and $10 \%$ CMS with $0.5 \mathrm{~Hz}$ mechanical stretching on the elastin, Lox and KDM4B protein levels in the BMSCs (Figure 1). The expression levels of elastin, LOX and KDM4B were increased with increased stretching, and the relative levels were significantly higher 
in the strained groups than in the unstrained control group $(P<0.05)$ (Figure 1a). In addition, the relative protein levels of elastin, LOX and KDM4B under 10\% CMS were obviously higher than those in the other groups $(P<0.05)$ (Figure 1b), which suggested that 10\% CMS was the optimal level for promoting BMSC differentiation into ligament fibroblasts.

\section{Influence of elastin and LOX on BMSCs after KDM4B knockdown or overexpression}

Based on the above observations, we hypothesized that $\mathrm{KDM} 4 \mathrm{~B}$ is required for the secretion of elastin and LOX by BMSCs. Therefore, to evaluate the role of KDM4B in the production of elastin and LOX in MSCs, we silenced or overexpressed KDM4B using shRNA vector constructs or lentiviral transduction, respectively, and we assessed whether the infection was successful in the cultured BMSCs. The results showed that the elastin and LOX protein levels were decreased after KDM4B shRNA transfection, suggesting that silencing KDM4B expression led to a loss of elastin and LOX in BMSCs, possibly through weakening the bonding at their promotor sites. For these results, significant differences were observed compared with both the non-targeted siRNA and negative control $(\mathrm{NC})$ groups $(P<0.05)$. However, overexpression of $\mathrm{KDM} 4 \mathrm{~B}$ in the BMSCs transiently transfected with pLVX-IRES-Puro-KDM4B obviously enhanced the rate of elastin and LOX secretion compared with that in the control vector and NC groups $(P<0.05)$ (Figure $2 \mathrm{a}$ and $2 \mathrm{~b}$ ). These results indicated that the production of elastin and LOX in the BMSCs was affected by the presence of KDM4B, which could promote BMSC differentiation by increasing the bonding to the start sites of the marker genes of ligament fibroblasts.

\section{Effect of H3K9me3 and KDM4B expression and binding to the LOX and elastin promotor at $10 \%$ CMS}

ChIP-PCR was performed to determine the histone methylation at regulatory regions for LOX and elastin in the BMSCs with 10\% CMS. The promoter sequences of the defined genes were analyzed by PCR amplification with the designed specific primers after immunoprecipitation of the chromatin. The results showed that $10 \%$ CMS promoted the binding of KDM4B to LOX and elastin, significantly upregulated the expression of LOX, and downregulated the expression of $\mathrm{H} 3 \mathrm{~K} 9 \mathrm{me} 3$. Moreover, depletion of KDM4B in addition to mechanical loading clearly decreased the binding ability of endogenous KDM4B to LOX and elastin compared with that in the NC group; however, the levels of KDM4B were significantly higher than that in the $0 \%$ CMS control group $(P<0.01)$ (Figure $3 \mathrm{~b}$ and $3 \mathrm{~d})$. In addition, the expression of H3K9me3 was significantly increased compared with that in the corresponding scramble control groups and was decreased compared with that in the scramble control groups under the NC condition $(P<0.01)$ (Figure $3 \mathrm{a}$ and $3 \mathrm{c})$; these findings indicated that mechanical stretching dramatically affected the activities of LOX and elastin by regulating the gene levels of $\mathrm{KDM} 4 \mathrm{~B}$ and $\mathrm{H} 3 \mathrm{~K} 9 \mathrm{me} 3$ in the promotor region. a

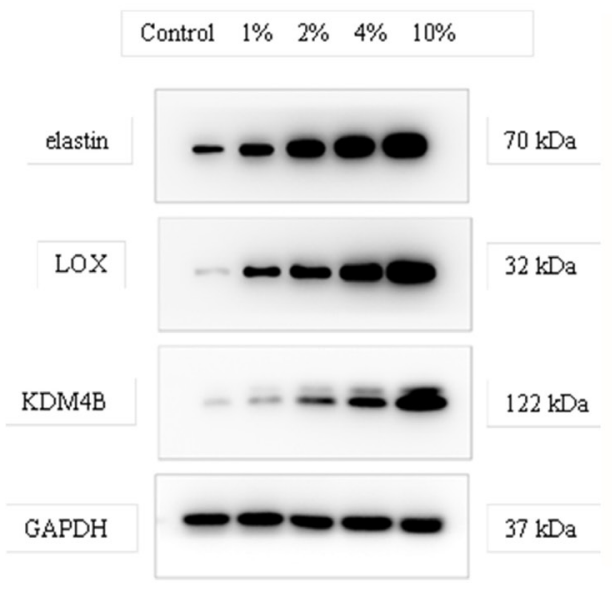

b

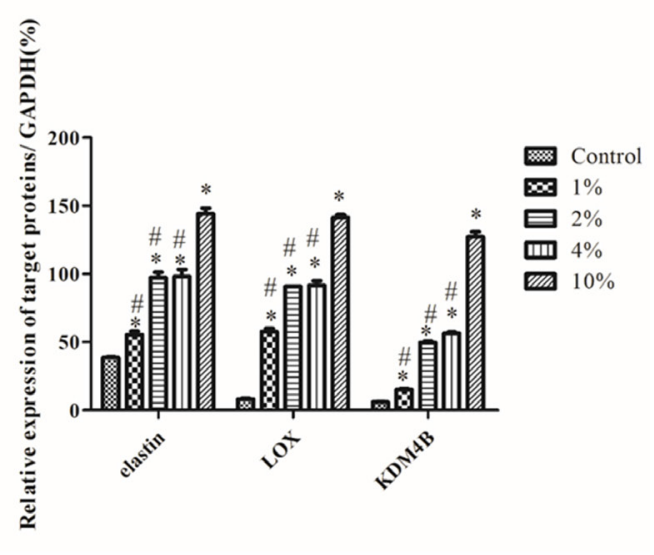

Figure 1: Elastin, LOX, and KDM4B protein levels in the BMSCs under stimulation with various amounts of stretch. (a) Induction of elastin, LOX and KDM4B with $0 \%$ (control), 1\%, 2\%, 4\% and 10\% CMS evaluated using Western blot assays. The band at $70 \mathrm{kDa}$ is elastin, the band at $32 \mathrm{kDa}$ is LOX, the band at $122 \mathrm{kDa}$ is KDM4B, and the band at $37 \mathrm{kDa}$ is GAPDH. (b) Protein levels of elastin, LOX, and KDM4B relative to GAPDH. This blot was based on three experiments. ${ }^{*} P<0.05$ vs. control group. ${ }^{\#} P<0.05$ vs. BMSC with $10 \%$ CMS. 
a

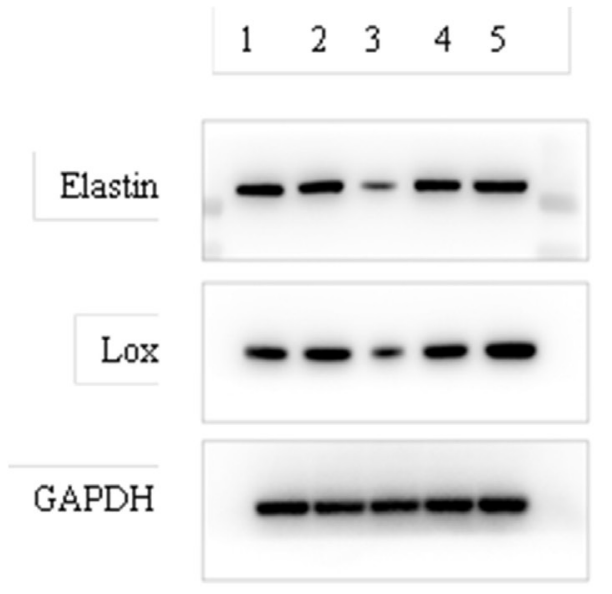

b

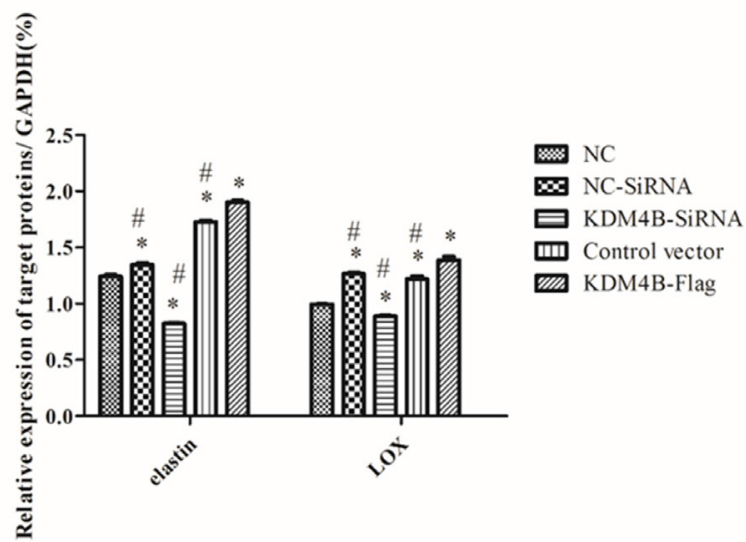

Figure 2: Depletion and overexpression of KDM4B affected the protein levels of elastin and Lox in BMSCs. (1) Negative control (NC), (2) non-targeted siRNA (NC-SiRNA), (3) KDM4B knockdown using siRNA (KDM4B-SiRNA), (4) empty vector used as a control, and (5) KDM4B overexpression (KDM4B-Flag). (a) Induction of elastin and LOX in the different groups according to the Western blot assay. (b) Protein levels of elastin and LOX relative to GAPDH. This blot was based on three experiments. ${ }^{*} P<0.05$ vs. the NC group. ${ }^{\#} P<0.05$ vs. the KDM4B-Flag group.

a

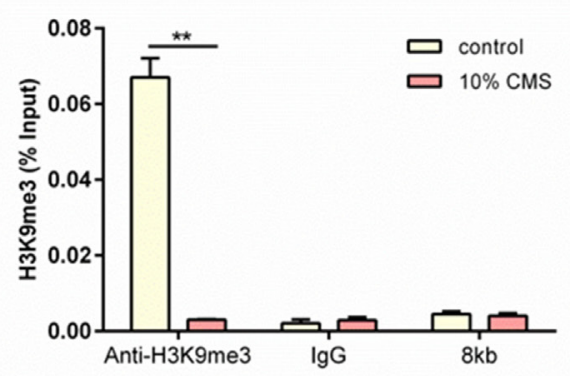

$\mathrm{C}$

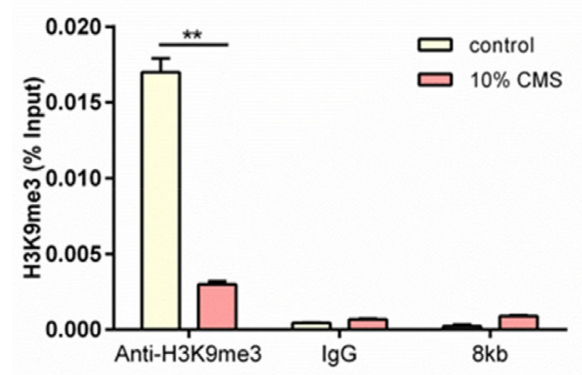

b

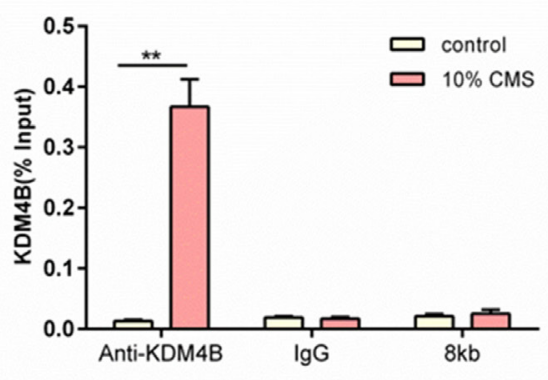

d

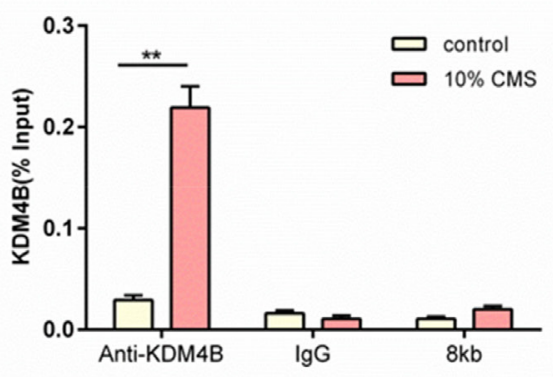

Figure 3: ChIP-PCR analysis of the effect of H3K9me3 and KDM4B expression on the LOX and elastin promoters in MSCs with 10\% CMS. The results were expressed relative to a 1/100 dilution of the chromatin inputs. (a) Relative effect of $\mathrm{H} 3 \mathrm{~K} 9 \mathrm{me} 3$ expression on elastin, (b) relative effect of KDM4B expression on elastin, (c) relative effect of H3K9me3 expression on LOX, and (d) relative effect of KDM4B expression on LOX. All results are representative of at least three independent experiments. ${ }^{* *} P<0.01$. The control group received $0 \%$ CMS. 


\section{Effect of H3K9me3 and KDM4B expression on LOX and elastin with 10\% CMS plus KDM4B knockdown}

We depleted KDM4B using KDM4B-targeted siRNA in BMSCs with $10 \%$ CMS and determined the expression of H3K9me3 and exogenous KDM4B in the promoter region of LOX and elastin in the BMSCs. The results showed that $10 \% \mathrm{CMS}$ could induce exogenous KDM4B expression $(P<0.01)$, and the KDM4B level in the scrambled-siRNA group was significantly higher than that of the KDM4B-siRNA group $(P<0.01)$ (Figure $4 \mathrm{~b}$ and $4 \mathrm{~d}$ ). However, 10\% CMS inhibited H3K9me3 expression compared with that in the $0 \%$ CMS control group $(P<0.01)$, although the $\mathrm{H} 3 \mathrm{~K} 9 \mathrm{me} 3$ level in the KDM4B-depleted group was significantly higher than that in the scrambled control group $(P<0.01)$ (Figure $4 \mathrm{a}$ and $4 \mathrm{c}$ ). These results indicate that $10 \% \mathrm{CMS}$ upregulated KDM4B expression but significantly downregulated H3K9me3 expression in the LOX and elastin promoter regions. Furthermore, in the CMS combined with KDM4B inhibition conditions, the exogenous KDM4B levels were

a

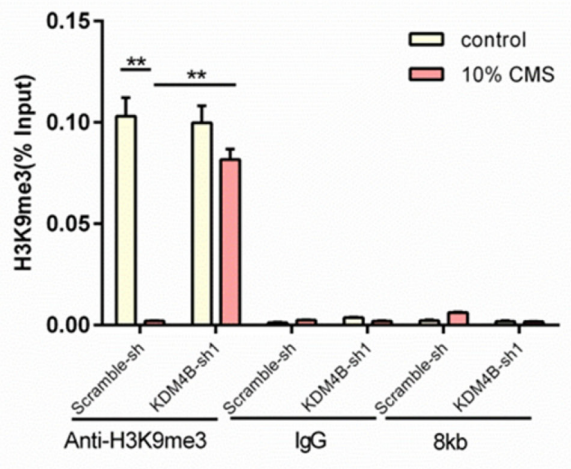

C

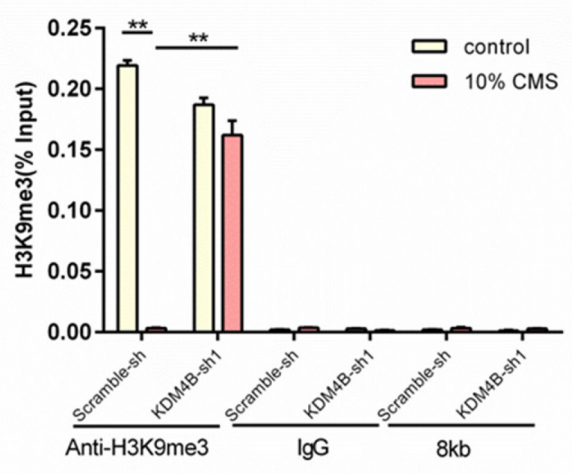

significantly decreased and the $\mathrm{H} 3 \mathrm{~K} 9 \mathrm{me} 3$ expression levels were significantly increased compared with those of the non-targeted scramble group.

\section{Immunofluoresence staining for the LOX protein in the BMSCs in vivo}

To further verify the effect of CMS on the regulation of the KDM4B and LOX protein expression in the BMSCs, we subcutaneously transplanted the BMSCs infected with the control (empty) vector, control vector $+10 \%$ CMS and KDM4B-Flag+10\% CMS with the amniotic membrane as a natural macromolecular material into mice and observed the positive expression of LOX via immunofluorescence assays. The results indicated an increase in the number of LOX-positive BMSCs in the KDM4B-Flag+10\% CMS group compared with the other two groups, with the lowest number of these cells in the empty vector group without cyclic force (Figure 5). These results demonstrated that the overexpression of exogenous KDM4B was beneficial for the production of LOX and further promoted the generation of ligament fibroblasts.

b
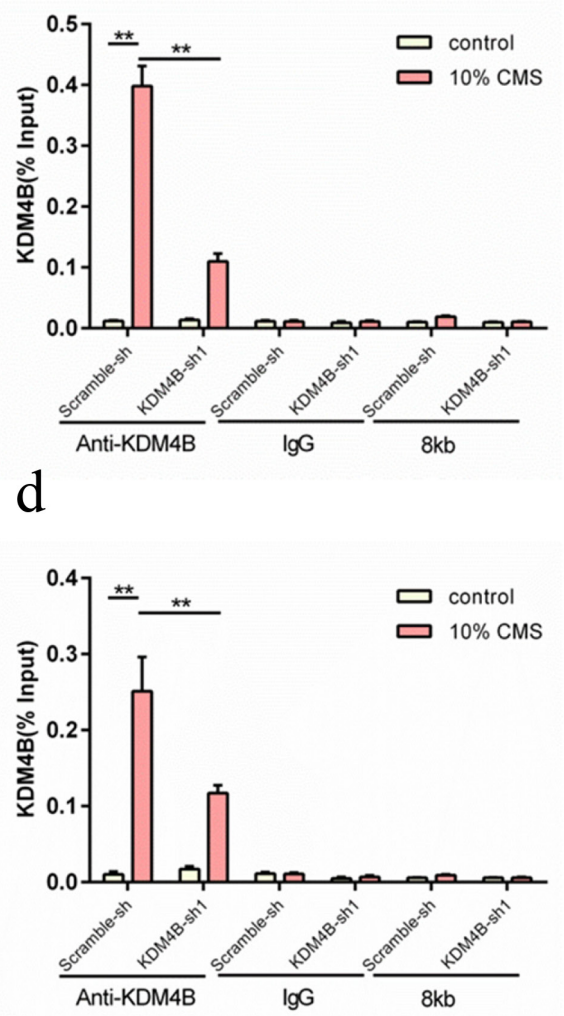

Figure 4: ChIP-PCR analysis of the effect of H3K9me3 and KDM4B expression on the regulatory regions of LOX and elastin in the MSCs subjected to $10 \%$ CMS plus KDM4B depletion. (a) Relative expression of H3K9me3 in the elastin promoter regions, (b) the relative expression of KDM4B in the elastin promoter regions, (c) the relative expression of H3K9me3 in the LOX promoter regions, and (d) the relative expression of KDM4B in the LOX promoter regions. ${ }^{* *} P<0.01$. The scrambled-siRNA group was the control group. 


\section{DISCUSSION}

PFD is closely related to the weakening of support and connective structures that results from ECM remodeling. Ligament fibroblasts are the main component of pelvic connective tissue, and they are considered a contributing factor in PFD [2-3]. Moreover, these fibroblasts are capable of synthesizing and secreting ECM proteins (collagen, elastin, and amino chitosan), and they are responsive to mechanical force and play important roles in wound healing and tissue reconstruction [12]. Elastin, a major ECM protein, elicits recoil in tissues and organs that undergo repeated stretch and breakdown and may initiate a powerful signal involving the whole wound repair pathway, including inflammation, repair and remodeling. Elastin damage may occur under the conditions of the excess mechanical and thermal forces that occur in several diseases, such as PFD. LOX is involved in the initiation of collagen synthesis and elastin cross-linkage, and it ensures that tropoelastin molecules remain highly crosslinked to one another, thus performing an indispensable role in the construction of connective tissue [29]. Yamamoto et al. [30] reported that the elastin expression level in the uterus was closely associated with POP, and the gene and protein levels of elastin were significantly decreased in the POP group compared with the control group. Liu et al. [31] found an increased incidence of POP in LOX gene knockout mice compared with the wild-type group during the postpartum period and found that the production of collagen and elastic fibers was inhibited after LOX gene knockout, resulting in PFD and lower urinary tract dysfunction. Jiang et al. [32] reported significantly lower expression of elastin and LOX family members in urogenital tissues in naturally and accelerated ovarian aging mice than in untreated young females, and this decrease caused the failure of elastic fiber synthesis and assembly and additional pelvic floor disorders.

Previous studies showed that CMS could modulate several cellular functions, such as cell proliferation, migration and paracrine signaling [33, 34]. The dynamic balance of connective tissue, such as tendons, ligaments, and pelvic connective tissues, requires the continuous application of biological force. Excessive tensile stress and cell deformation frequencies affect the cell adhesion strength and can damage the cells of the cytoskeleton, and they also increase the complexity of the cell mechanical environment, which reduces the ability to control cell conditions experimentally. However, at inadequate frequencies, the cell conditions cannot be fully simulated [35]. In this study, conditions of $1 \%, 2 \%$,
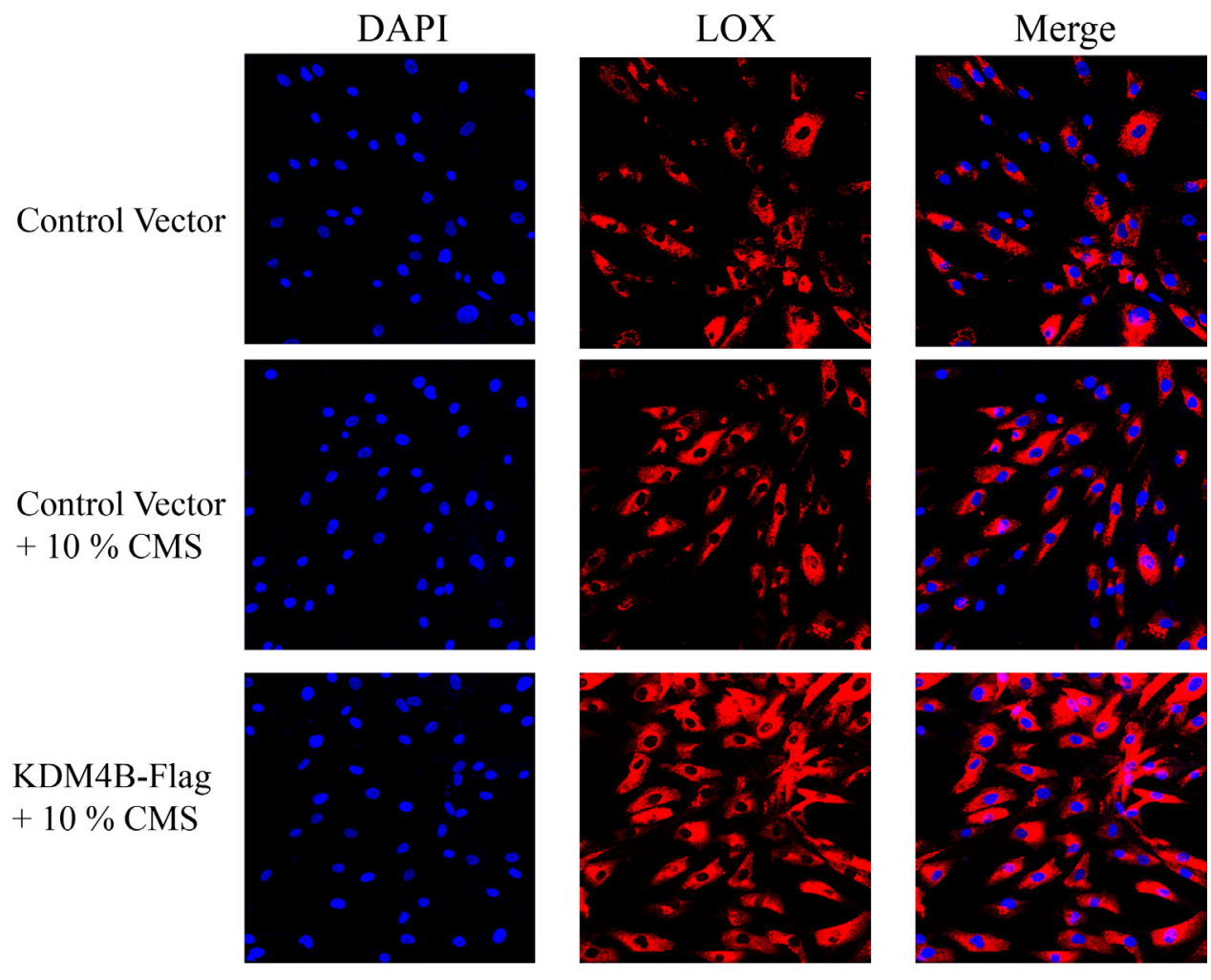

Figure 5: Immunofluoresence staining for the LOX protein in the BMSCs in vivo (400×). BMSCs were infected with lentiviruses designed to express KDM4B. DAPI staining was used to indicate the location of the nucleus. Empty vectors (pLVX-IRESPuro) were used as a control. 
$4 \%$ and $10 \%$ CMS at a $0.5 \mathrm{~Hz}$ frequency were applied to the BMSCs to induce changes in LOX and elastin expression, and the results showed that the mechanical stimulation facilitated the expression of LOX and elastin, whose levels were consistently increased in our system in response to the continuous application of a mechanical load. Moreover, the $10 \%$ CMS condition best stimulated the BMSC growth. Zhu et al. [36] found that mechanical stretching with $10 \%$ extension significantly increased the angiogenic capacity of MSCs. Our previous study also confirmed that $10 \%$ mechanical stretching could promote BMSC differentiation in an indirect co-culture system with pelvic ligament fibroblasts, as indicated by the increased expression of elastin and LOX.

We hypothesized that the changes in the LOX and elastin expression after mechanical stimulation were closely related to histone modifications. In this study, we found that KDM4B could directly or indirectly bind to the promoter region of LOX and elastin, and its expression in the BMSCs was induced in response to additional stretching. In addition, 10\% CMS and a $0.5 \mathrm{~Hz}$ frequency were the optimal conditions for inducing the secretion of KDM4B in the BMSCs, and a similar trend was observed for the LOX and elastin expression. Overexpression of KDM4B significantly increased the protein levels of elastin and LOX in the BMSCs compared with those in the control vector group. Similarly, the immunofluorescence assays revealed that the overexpression of KDM4B and the application of $10 \%$ CMS increased the number of LOX-positive cells, which were associated with the production of ligament fibroblasts in vivo. Conversely, the depletion of KDM4B significantly decreased the protein levels of elastin and LOX in the BMSCs, suggesting that KDM4B was required for the BMSC differentiation into ligament fibroblasts. Ye et al. reported that the depletion of KDM4B significantly reduced the osteogenic differentiation of MSCs in vitro and in vivo [37]. Lee et al. also reported that KDM4B overexpression significantly enhanced the chondrogenic differentiation and that KDM4B depletion using shRNA significantly reduced the chondrogenic potential [38].

KDM4B enzymes are histone demethylases with specificity for $\mathrm{H} 3 \mathrm{~K} 9 \mathrm{me} 3$, which is associated with transcriptional repression when localized to transcription start sites [39]. The ChIP-PCR assay revealed that the effect of KDM4B on the LOX and elastin promoter regions under 10\% CMS was increased compared with that of the $0 \% \mathrm{CMS}$ control group regardless of whether KDM4B was silenced in the BMSCs, and the opposite results were observed for $\mathrm{H} 3 \mathrm{~K} 9 \mathrm{me} 3$. This finding indicated that CMS could improve the ability of KDM4B to combine with LOX and elastin in the BMSCs, which is associated with the downregulation of $\mathrm{H} 3 \mathrm{~K} 9 \mathrm{me} 3$. Our results provide the first demonstration that $10 \%$ CMS could promote the differentiation of BMSCs into ligament fibroblasts via LOX and elastin activation because of the epigenetic induction of KDM4B expression.

\section{MATERIALS AND METHODS}

\section{Cell culture}

Mouse femurs were rinsed with $\alpha$-MEM medium, and the bone marrow rinse fluid was cultured in $\alpha$-MEM with $10 \%$ fetal bovine serum (FBS) and 1\% penicillin and streptomycin. The culture conditions were $37^{\circ} \mathrm{C}$ and $5 \% \mathrm{CO}_{2}$. After 3 days of culture, non-adherent cells were removed. The adherent cultured BMSCs were subcultured until passages 3 to 7 for this experimental study.

\section{Lentiviral transduction overexpression studies}

KDM4B cDNA was cloned into a pLVX-IRESpuro overexpression vector to construct a KDM4B overexpression plasmid. The pLVX-IRES-puro and pLVX-IRES-puro-KDM4B overexpression plasmids were transfected with psPAX2 and pMD2.G into HEK293 cells. Forty-eight hours after transfection, the virus supernatant was collected to infect BMSCs.

\section{shRNA knockdown studies}

The pLKO.1-KDM4B lentiviral shRNA vectors that target KDM4B and pLKO.1 control vectors (Scrsh) were synthesized by Open Biosystems (Thermo Fisher Scientific, Inc.). The pLKO.1-KDM4B lentiviral shRNA vectors and pLKO.1 control vectors (Scrsh) were transfected with psPAX2 and pMD2.G into HEK293 cells. Forty-eight hours after transfection, the virus supernatant was collected to infect MSCs.

\section{Western blot analysis}

Cells were harvested and lysed on ice using a lysis buffer for 30 minutes. The protein lysate was collected by centrifugation at $15,000 \mathrm{~g}$ for 10 minutes at $4^{\circ} \mathrm{C}$. The cell lysates were subjected to gel electrophoresis and then transferred to PVDF membranes, which were blocked with $5 \% \mathrm{BSA}$ for $1 \mathrm{~h}$ and incubated overnight with specific antibodies at $4^{\circ} \mathrm{C}$. This step was followed by incubation with horseradish peroxidase-labeled secondary antibody for one hour. The following primary antibodies were used in this study: anti-KDM4B, anti-Elastin, anti-LOX pAb (1:1000, Abcam, Cambridge, UK), and anti-GAPDH mAb (1:1000, Cell Signaling Technology, Inc.).

\section{Cyclic mechanical stretch application}

Mechanical stimulation was initiated when the cell density reached $50-60 \%$. CMS with a $0.5-\mathrm{Hz}$ sinusoidal curve at $10 \%$ elongation ( $8 \mathrm{~h}$ per day for 1 weeks) 
was applied using an FX-5000 T Flexcell Tension Plus unit (Flexcell International Corporation). The culture conditions were $37^{\circ} \mathrm{C}$ and $5 \% \mathrm{CO}_{2}$ in a humidified atmosphere. Cells were harvested immediately after the application of CMS stimulation was completed. The control cells were seeded in the same culture plate, and the culture conditions were the same. The cells were harvested immediately after the end of the mechanical stimulation.

\section{ChIP assay}

ChIP assays were performed using an EZ ChIP Chromatin Immunoprecipitation Kit (Millipore, Upstate) according to the manufacturer's instructions. Briefly, the cells were fixed at room temperature using a $1 \%$ formic acid solution for 10 minutes. Next, the cells were washed twice with PBS, placed in lysis buffer containing protease inhibitors, resuspended and then sonicated. DNA fragments from the soluble chromatin preparations were 400-800 $\mathrm{bp}$ in length. Immunoprecipitation (IP) was carried out overnight with purified anti-H3K9me3 and anti-SMAD5 antibodies (Millipore, Bedford, MA, USA) or normal mouse IgG as a negative control. Protein $\mathrm{A} / \mathrm{G}$ agarose was used to pull down the antigen-antibody compounds, and then the sample was washed four times with washing buffers. The DNA-protein crosslinks were reversed using $5 \mathrm{M} \mathrm{NaCl}$ at $65^{\circ} \mathrm{C}$ for $6 \mathrm{~h}$, and DNA from each sample was purified. PCR was performed using $2 \mu$ of the DNA samples with the following primers. Elastin primer: forward, 5'-GATGGCGGGTCTGACAGCGGTAGT-3'; reverse, 5'-GATGGAGGAGGTTGAGCA AGAGGAT-3'; $8 \mathrm{~kb}$ primer: forward, 5'AGTCCATTGACCTTATGATCCAAC-3'; reverse, 5'-AATCGAAAATCGATTCGTCGTAT-3'. LOX primer: forward, 5'-ATGGTGCTCCCGGCTCGTCCCTTCT-3'; reverse, 5' - AACTGCAAACTGCCACGTCCTCC-3'; 8 kb primer: forward, 5'- TCTTCCACTCGGTGCGTCT -3'; reverse, 5' - ATGTTCCCTGGGTAGGTAACTC -3'.

\section{Immunofluorescence}

Nude mouse were randomly divided into three groups, each containing 6 mice. BMSCs (Vector, Vector $+\mathrm{CMS}$, Vector + CMS + KDM4B) at passage 3 were resuspended in a mixture of medium and Matrigel (BD Bioscience) and then transplanted subcutaneously. Two weeks after transplantation, $5 \mu \mathrm{m}$ tissue sections were frozen. After two rinses with PBS, the membrane was permeabilized using $0.5 \%$ Triton $\mathrm{X}-100$ for 5 minutes and blocked with $5 \%$ BSA for 60 minutes. The sample was incubated with primary anti-LOX antibodies (Abcam, Cambridge, UK) at $4^{\circ} \mathrm{C}$ overnight and then incubated with PE-conjugated anti-rabbit IgG secondary antibodies. After two washes with PBS, the nuclei were counterstained with DAPI for 10 minutes. The tissue staining was detected using laser confocal microscopy.

\section{Statistical analysis}

SPSS analytics software (version 11.5, SPSS Inc., Chicago, IL, USA) was used for the statistical analyses. The results are presented as the mean \pm standard deviation (SD) and were analyzed using a one-way analysis of variance (ANOVA). The gene expression levels relative to the GAPDH expression were calculated according to the $2^{-\Delta \Delta \mathrm{Ct}}$ method. $P<0.05$ was considered statistically significant.

\section{CONCLUSION}

We have provided evidence that $10 \%$ CMS at a 0.5 $\mathrm{Hz}$ frequency could enhance the directed differentiation of BMSCs to ligament fibroblasts via the positive regulation of LOX and elastin because these conditions induce the secretion of KDM4B while inhibiting the expression of H3K9me3. Our experiments represent a novel approach to promoting the production of ligament fibroblasts that could be used in stem cell therapies for PFD.

\section{Abbreviations}

PFD: Pelvic floor dysfunction; SUI: Stress urinary incontinence; POP: Pelvic organ prolapse; BMSCs: Bone mesenchymal stem cells; CMS: cyclic mechanical stretch; FI: fecal incontinence; LOX: Lysyl oxidase; ECM: Extracellular matrix components; ChIP-PCR: Chromatin immunoprecipitation PCR.

\section{Author contributions}

Bing Zhao, Junmin Wang and Shihong Cui conceived and designed the study. Shenshen Ren, Mengcai $\mathrm{Hu}$, Huiyan $\mathrm{Wu}$, Lulu Chen, Feng Xu, Xueqin Zheng and Juan Chen performed the experiments. Xiaojun Liu cultured cells and provided the HEK293T cells and pLVXIRES- Puro vector. Junmin Wang and Bing Zhao wrote the paper. Shenshen Ren, Mengcai Hu, Huiyan Wu and Lulu Chen reviewed and edited the manuscript. All authors read and approved the manuscript.

\section{ACKNOWLEDGMENTS}

We appreciate all the other staff members of the Laboratory Animal Center of Zhengzhou University for providing assistance with the experiments.

\section{CONFLICTS OF INTEREST}

All of the authors indicated no potential conflicts of interest. 


\section{FUNDING}

This work was supported by the Natural Science Foundation of China (Grant Nos. 81300469 and 31201945) and the Medical Science Research Project of Henan Province (No. 21303093).

\section{REFERENCES}

1. Perry S, Shaw C, Assassa P, Dallosso H, Williams K, Brittain KR, Mensah F, Smith N, Clarke M, Jagger C, Mayne C, Castleden CM, Jones J, McGrother C, and Leicestershire MRC Incontinence Study Team. An epidemiological study to establish the prevalence of urinary symptoms and felt need in the community: the Leicestershire MRC Incontinence Study. J Public Health Med. 2000; 22:427-34. https://doi.org/10.1093/ pubmed/22.3.427.

2. Wen Y, Wani P, Zhou L, Baer T, Phadnis SM, Reijo Pera RA, Chen B. Reprogramming of fibroblasts from older women with pelvic floor disorders alters cellular behavior associated with donor age. Stem Cells Transl Med. 2013; 2:118-28. https://doi.org/10.5966/sctm.2012-0092.

3. Mangera A, Bullock AJ, Roman S, Chapple CR, MacNeil S. Comparison of candidate scaffolds for tissue engineering for stress urinary incontinence and pelvic organ prolapse repair. BJU Int. 2013; 112:674-85. https://doi.org/10.1111/ bju.12186.

4. Mangir N, Bullock AJ, Roman S, Osman N, Chapple C, MacNeil S. Production of ascorbic acid releasing biomaterials for pelvic floor repair. Acta Biomater. 2016; 29:188-97. https://doi.org/10.1016/j.actbio.2015.10.019.

5. Rortveit G, Daltveit AK, Hannestad YS, Hunskaar S, and Norwegian EPINCONT Study. Urinary incontinence after vaginal delivery or cesarean section. N Engl J Med. 2003; 348:900-07. https://doi.org/10.1056/NEJMoa021788.

6. Lee JH, Wen Y, Polan ML, Chen B. The effect of raloxifene, a SERM, on extracellular matrix protein expression of pelvic fibroblasts. Int Urogynecol J Pelvic Floor Dysfunct. 2012; 23:349-55. https://doi.org/10.1007/ s00192-011-1567-0.

7. Awad HA, Butler DL, Boivin GP, Smith FN, Malaviya P, Huibregtse B, Caplan AI. Autologous mesenchymal stem cell-mediated repair of tendon. Tissue Eng. 1999; 5:267-77. https://doi.org/10.1089/ten.1999.5.267.

8. Omoto M, Miyashita H, Shimmura S, Higa K, Kawakita T, Yoshida S, McGrogan M, Shimazaki J, Tsubota K. The use of human mesenchymal stem cell-derived feeder cells for the cultivation of transplantable epithelial sheets. Invest Ophthalmol Vis Sci. 2009; 50:2109-15. https://doi. org/10.1167/iovs.08-2262.

9. Watanabe N, Woo SL, Papageorgiou C, Celechovsky C, Takai S. Fate of donor bone marrow cells in medial collateral ligament after simulated autologous transplantation. Microsc Res Tech. 2002; 58:39-44. https:// doi.org/10.1002/jemt.10115.

10. Young RG, Butler DL, Weber W, Caplan AI, Gordon SL, Fink DJ. Use of mesenchymal stem cells in a collagen matrix for Achilles tendon repair. J Orthop Res. 1998; 16:406-13. https://doi.org/10.1002/jor.1100160403.

11. Smith AN, Willis E, Chan VT, Muffley LA, Isik FF, Gibran NS, Hocking AM. Mesenchymal stem cells induce dermal fibroblast responses to injury. Exp Cell Res. 2010; 316:4854. https://doi.org/10.1016/j.yexcr.2009.08.001.

12. Ozsvar J, Mithieux SM, Wang R, Weiss AS. Elastin-based biomaterials and mesenchymal stem cells. Biomater Sci. 2015; 3:800-09. https://doi.org/10.1039/C5BM00038F.

13. Çelebi B, Cloutier M, Rabelo RB, Mantovani D, Bandiera A, Bandiera A. Human elastin-based recombinant biopolymers improve mesenchymal stem cell differentiation. Macromol Biosci. 2012; 12:1546-54. https://doi.org/10.1002/mabi.201200170.

14. Ruiz LA, Báez-Vega PM, Ruiz A, Peterse DP, Monteiro JB, Bracero N, Beauchamp P, Fazleabas AT, Flores I. Dysregulation of lysyl oxidase expression in lesions and endometrium of women with endometriosis. Reprod Sci. 2015; 22:1496-508. https://doi. org/10.1177/1933719115585144.

15. El-Haibi CP, Bell GW, Zhang J, Collmann AY, Wood D, Scherber CM, Csizmadia E, Mariani O, Zhu C, Campagne A, Toner M, Bhatia SN, Irimia D, et al. Critical role for lysyl oxidase in mesenchymal stem cell-driven breast cancer malignancy. Proc Natl Acad Sci USA. 2012; 109:17460-65. https://doi.org/10.1073/pnas.1206653109.

16. Martin C, Zhang Y. The diverse functions of histone lysine methylation. Nat Rev Mol Cell Biol. 2005; 6:838-49. https://doi.org/10.1038/nrm1761.

17. Jack AP, Bussemer S, Hahn M, Pünzeler S, Snyder M, Wells M, Csankovszki G, Solovei I, Schotta G, Hake SB. H3K56me3 is a novel, conserved heterochromatic mark that largely but not completely overlaps with $\mathrm{H} 3 \mathrm{~K} 9 \mathrm{me} 3$ in both regulation and localization. PLoS One. 2013; 8:e51765. https://doi.org/10.1371/journal.pone.0051765.

18. Labbé RM, Holowatyj A, Yang ZQ. Histone lysine demethylase (KDM) subfamily 4: structures, functions and therapeutic potential. Am J Transl Res. 2013; 6:1-15.

19. Das ND, Choi MR, Jung KH, Park JH, Lee HT, Das A, Kim $\mathrm{SH}$, Chai YG. Functional analysis of histone demethylase $\mathrm{Jmjd} 2 \mathrm{~b}$ on lipopolysaccharide-treated murine neural stem cells (NSCs). Neurotox Res. 2013; 23:154-65. https://doi. org/10.1007/s12640-012-9346-3.

20. Uribe RA, Buzzi AL, Bronner ME, Strobl-Mazzulla $\mathrm{PH}$. Histone demethylase KDM4B regulates otic vesicle invagination via epigenetic control of Dlx3 expression. J Cell Biol. 2015; 211:815-27. https://doi.org/10.1083/ jcb.201503071.

21. Young LC, Hendzel MJ. The oncogenic potential of Jumonji D2 (JMJD2/KDM4) histone demethylase overexpression. 
Biochem Cell Biol. 2013; 91:369-77. https://doi. org/10.1139/bcb-2012-0054.

22. Zhao L, Li W, Zang W, Liu Z, Xu X, Yu H, Yang Q, Jia J. JMJD2B promotes epithelial-mesenchymal transition by cooperating with $\beta$-catenin and enhances gastric cancer metastasis. Clin Cancer Res. 2013; 19:6419-29. https://doi. org/10.1158/1078-0432.CCR-13-0254.

23. Kim TJ, Sun J, Lu S, Qi YX, Wang Y. Prolonged mechanical stretch initiates intracellular calcium oscillations in human mesenchymal stem cells. PLoS One. 2014; 9:e109378. https://doi.org/10.1371/journal.pone.0109378.

24. Hao J, Zhang Y, Jing D, Shen Y, Tang G, Huang S, Zhao Z. Mechanobiology of mesenchymal stem cells: perspective into mechanical induction of MSC fate. Acta Biomater. 2015; 20:1-9. https://doi.org/10.1016/j.actbio.2015.04.008.

25. Martins RP, Finan JD, Guilak F, Lee DA. Mechanical regulation of nuclear structure and function. Annu Rev Biomed Eng. 2012; 14:431-55. https://doi.org/10.1146/ annurev-bioeng-071910-124638.

26. Obi S, Yamamoto K, Ando J. Effects of shear stress on endothelial progenitor cells. J Biomed Nanotechnol. 2014; 10:2586-97. https://doi.org/10.1166/jbn.2014.2014.

27. Zhang B, Luo Q, Chen Z, Sun J, Xu B, Ju Y, Song G. Cyclic mechanical stretching promotes migration but inhibits invasion of rat bone marrow stromal cells. Stem Cell Res (Amst). 2015; 14:155-64. https://doi.org/10.1016/j. scr.2015.01.001.

28. Bing Z, Linlin L, Jianguo Y, Shenshen R, Ruifang R, Xi Z. Effect of mechanical stretch on the expressions of elastin, LOX and Fibulin-5 in rat BMSCs with ligament fibroblasts co-culture. Mol Biol Rep. 2012; 39:6077-85. https://doi. org/10.1007/s11033-011-1422-X.

29. Park SY, Park JC, Kim MS, Lee SE, Kim KJ, Jung BJ, Park W, Jeon DW, Cho KS, Kim CS. Differential effect of water-soluble chitin on collagen synthesis of human bone marrow stem cells and human periodontal ligament stem cells. Tissue Eng Part A. 2015; 21:451-62. https://doi. org/10.1089/ten.tea.2014.0156.

30. Yamamoto M, Akazawa K, Aoyagi M, Yamamoto N, Yamamoto K. Changes in elastin-binding protein in fibroblasts derived from cardinal ligaments of patients with prolapsus uteri. Cell Biol Int. 2002; 26:441-49. https://doi. org/10.1006/cbir.2002.0877.
31. Liu G, Daneshgari F, Li M, Lin D, Lee U, Li T, Damaser MS. Bladder and urethral function in pelvic organ prolapsed lysyl oxidase like-1 knockout mice. BJU Int. 2007; 100:41418. https://doi.org/10.1111/j.1464-410X.2007.06929.x.

32. Jiang Y, Zong W, Luan H, Liu JH, Zhang AZ, Li XL, Liu SY, Zhang SQ, Gao JG. Decreased expression of elastin and lysyl oxidase family genes in urogenital tissues of aging mice. J Obstet Gynaecol Res. 2014; 40:1998-2004. https:// doi.org/10.1111/jog.12425.

33. Egginton S. Physiological factors influencing capillary growth. Acta Physiol (Oxf). 2011; 202:225-39. https://doi. org/10.1111/j.1748-1716.2010.02194.x.

34. Kang MN, Yoon HH, Seo YK, Park JK. Effect of mechanical stimulation on the differentiation of cord stem cells. Connect Tissue Res. 2012; 53:149-59. https:// doi.org/10.3109/03008207.2011.619284.

35. Horikawa A, Okada K, Sato K, Sato M. Morphological changes in osteoblastic cells (MC3T3-E1) due to fluid shear stress: cellular damage by prolonged application of fluid shear stress. Tohoku J Exp Med. 2000; 191:127-37. https:// doi.org/10.1620/tjem.191.127.

36. Zhu Z, Gan X, Fan H, Yu H. Mechanical stretch endows mesenchymal stem cells stronger angiogenic and antiapoptotic capacities via NFKB activation. Biochem Biophys Res Commun. 2015; 468:601-05. https://doi.org/10.1016/j. bbrc.2015.10.157.

37. Ye L, Fan Z, Yu B, Chang J, Al Hezaimi K, Zhou X, Park $\mathrm{NH}$, Wang CY. Histone demethylases KDM4B and KDM6B promotes osteogenic differentiation of human MSCs. Cell Stem Cell. 2012; 11:50-61. https://doi.org/10.1016/j. stem.2012.04.009.

38. Lee HL, Yu B, Deng P, Wang CY, Hong C. Transforming growth factor- $\beta$-induced KDM4B promotes chondrogenic differentiation of human mesenchymal stem cells. Stem Cells. 2016; 34:711-19. https://doi.org/10.1002/stem.2231.

39. Iwamori N, Zhao M, Meistrich ML, Matzuk MM. The testis-enriched histone demethylase, KDM4D, regulates methylation of histone $\mathrm{H} 3$ lysine 9 during spermatogenesis in the mouse but is dispensable for fertility. Biol Reprod. 2011; 84:1225-34. https://doi.org/10.1095/ biolreprod.110.088955. 